\title{
Transmitted Waveform Design Based on Iterative Methods
}

\author{
Bin Wang, ${ }^{1}$ Jinkuan Wang, ${ }^{1}$ Xin Song, ${ }^{1}$ and Fengming Xin ${ }^{2}$ \\ ${ }^{1}$ College of Computer and Communication Engineering, Northeastern University at Qinhuangdao, Qinhuangdao 066004, China \\ ${ }^{2}$ College of Information Science and Engineering, Northeastern University, Shenyang 110004, China \\ Correspondence should be addressed to Bin Wang; wangbin_neu@yahoo.com.cn
}

Received 24 October 2012; Revised 9 January 2013; Accepted 24 January 2013

Academic Editor: Alicia Cordero

Copyright (C) 2013 Bin Wang et al. This is an open access article distributed under the Creative Commons Attribution License, which permits unrestricted use, distribution, and reproduction in any medium, provided the original work is properly cited.

\begin{abstract}
In intelligent radar, it is an important problem for the transmitted waveform to adapt to the environment in which radar works. In this paper, we propose mutual information model of adaptive waveform design, which can convert the problem of adaptive waveform design into the problem of optimization. We consider two situations of no clutter and clutter and use Newton method and interior point method to solve the optimization problem. Then we can draw the design criterion for the transmitted waveform in cognitive radar and get a greater mutual information from the simulation results. Finally, the whole paper is summarized.
\end{abstract}

\section{Introduction}

The word radar is an acronym for radio detection and ranging. Today, the technology is so common that the word has become a standard English noun. The history of radar extends to the early days of modern electromagnetic theory. Radar is an electromagnetic system for the detection and location of reflecting objects such as aircraft, ships, spacecraft, vehicles, people, and the natural environment. It is widely used for surveillance, tracking, and imaging applications, for both civilian and military needs. Early radar development was driven by military necessity, and nowadays the military is still the dominant user and developer of radar technology. All early radars use radio waves, but some modern radar today are based on optical waves and the use of lasers. Radar development was accelerated during World War II. Since that time development has continued, such that present-day systems are very sophisticated and advanced. However, traditional radar systems are lack of adaptivity to the environment in which it works. Now the radar working conditions are more and more complex. Modern radar systems should transmit different waveforms according to different environment. So we need to consider the problem of adaptive waveform design.

Cognitive radar is a new framework of radar system proposed by Haykin [1] in 2006. Cognitive radar is an advanced form of radar system and it may adaptively and intelligently interrogate a propagation channel using all available knowledge including previous measurements, task priorities, and external databases. In cognitive radar, the radar continuously learns about the environment through experience gained from interactions of the receiver with the environment, the transmitter adjusts its illumination of the environment in an intelligent manner and the whole radar system constitutes a closed-loop dynamic system. There are three basic ingredients in the composition of cognitive radar: Intelligent signal processing, which itself builds on learning through interactions of the radar with the surrounding environment; Feedback from the receiver to the transmitter, which is a facilitator of intelligence; Preservation of the information content of radar returns, which is realized by the Bayesian approach to radar signal processing. Haykin [2] suggests that such a cognitive radar system can be represented using a Bayesian formulation whereby many different channel hypotheses are given a probabilistic rating. As more information is collected, the parameters of the channel hypotheses and their relative likelihoods are updated. The goal of an illumination, therefore, is to efficiently reduce the uncertainty attributed to each channel hypothesis. Hard decisions are only made when confidence is sufficient or when necessity mandates an immediate action. In 2009, Simon Haykin in another paper introduces the realization methods of cognitive radar. He suggests that to sense the radar environment, the receiver uses approximate Bayesian filtering and to control the radar illumination, the transmitter uses an incremental dynamic programming. Arasaratnam and 
Haykin [3] have successfully solved the best approximation to the Bayesian filter in the sense of completely preserving second-order information, which is called Cubature Kalman filters. Haykin et al. [4] propose a waveform design method that efficiently synthesizes waveforms that provide a tradeoff between estimation performance for a Gaussian ensemble of targets and detection performance for a specific target. Yang and Blum [5] address the problem of optimum radar waveform design for both radars employing a single transmit and receive antenna and the recently proposed multiple-input multiple-output radar. Goodman et al. [6] compare two different waveform design techniques for use with active sensors operating in a target recognition application and proposes the integration of waveform design with a sequential-hypothesistesting framework that controls when hard decisions may be made with adequate confidence. Sira et al. [7] consider joint sensor configuration and tracking for the problem of tracking a single target in the presence of clutter using range and range-rate measurements obtained by waveform-agile, active sensors in a narrowband environment. An algorithm to select and configure linear and nonlinear frequencymodulated waveforms is then proposed. Yang and Blum [8] use a random target impulse response to model the scattering characteristics of the extended (nonpoint) target, and two radar waveform design problems with constraints on waveform power have been investigated. Leshem et al. [9] describe the optimization of an information theoretic criterion for radar waveform design. Romero and Goodman [10] present illumination waveforms matched to stochastic targets in the presence of signal-dependent interference. The waveforms are formed by SNR and MI optimization. Kwon [11] presents waveform design methods for piezo inkjet dispensers based on measured meniscus motion. Kershaw and Evans [12] present an adaptive, waveform selective probabilistic data association (WSPDA) algorithm for tracking a single target in clutter. Sira and Cochran [13] propose a method to employ waveform agility to improve the detection of low radarcross section (RCS) targets on the ocean surface that present low signal-to-clutter ratios due to high sea states and low grazing angles. Rago et al. [14] investigate the performance of combined constant and swept frequency waveform fusion systems. The results indicate that the overall detectiontracking performance is strongly dependent on the waveform used, and that the use of the optimal waveform can lead to dramatic improvement in tracking error.

In this paper, we propose mutual information model of adaptive waveform design, which can convert the problem of adaptive waveform design into the problem of optimization. We consider two situations of no clutter and clutter and use Newton method and interior point method to solve the optimization problem. Then we can draw the design criterion for the transmitted waveform in cognitive radar from the simulation results.

\section{Mutual Information Model of Adaptive Waveform Design}

The basic parts of a radar system are illustrated in the diagram of Figure 1. The equipment is divided into several subsystems,

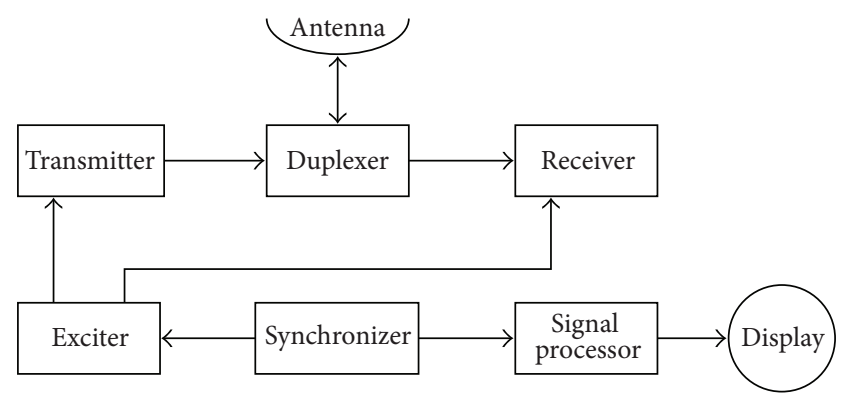

FIgURE 1: Block diagram of a typical radar system.

corresponding to the usual design specialties within the radar engineering field. A radar system has a transmitter that emits radio waves called radar signals in predetermined directions. When they come into contact with an object the signals are usually reflected and/or scattered in many directions. The radar signals that are reflected back towards the transmitter are the desirable ones which make radar work.

Different from traditional radar, cognitive radar is constructed using intelligent signal processing, information feedback loop, and soft information processing. In cognitive radar, the radar continuously learns about the environment through experience gained from interactions of the receiver with the environment, the transmitter adjusts its illumination of the environment in an intelligent manner, and the whole radar system constitutes a closed-loop dynamic system. Figure 2 is the block diagram of cognitive radar [1].

From the block diagram of cognitive radar, we can see that constructing a waveform library is very important in cognitive radar. Through sensing the environment, cognitive radar transmits waveform suited to the working conditions. The radar returns, and environment factors can help to reconstruct the waveform library. Then the radar can select different waveforms to transmit. It forms a feedback loop, and the cycle goes on and on. Figure 3 is block diagram of waveform library.

Waveform library can store many kinds of waveforms. The design of radar waveforms has been a topic of considerable research interest for several decades. In traditional radar systems, the radar transmits single waveform. The radar is difficult to adapt to different environments. Modern radar is required to transmit different waveforms according to different environments. So a more flexible design framework is required, which should be able to synthesize waveforms that provide a smooth trade-off between competing design criteria.

Cognitive radar is the next generation radar system. Figure 4 is a basic signal-processing cycle in cognitive radar. Cognitive radar has the capability to observe and learn from the environment. It operates in closed loop, and the transmitted waveform will be adaptive. In order to achieve objectives more efficiently, the waveforms should be adapted in response to prior measurements.

Generally speaking, the waveform design is different as a result of different tasks of radar. For detection task, the optimal radar waveform should be able to put as much 


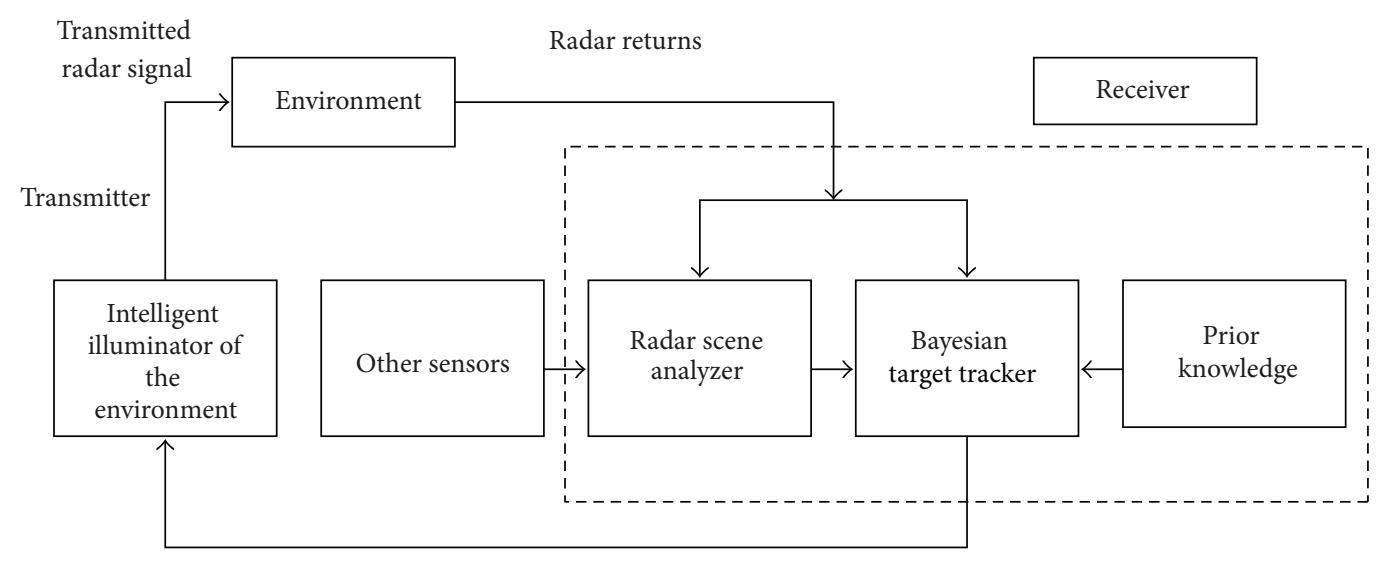

Figure 2: Block diagram of cognitive radar.

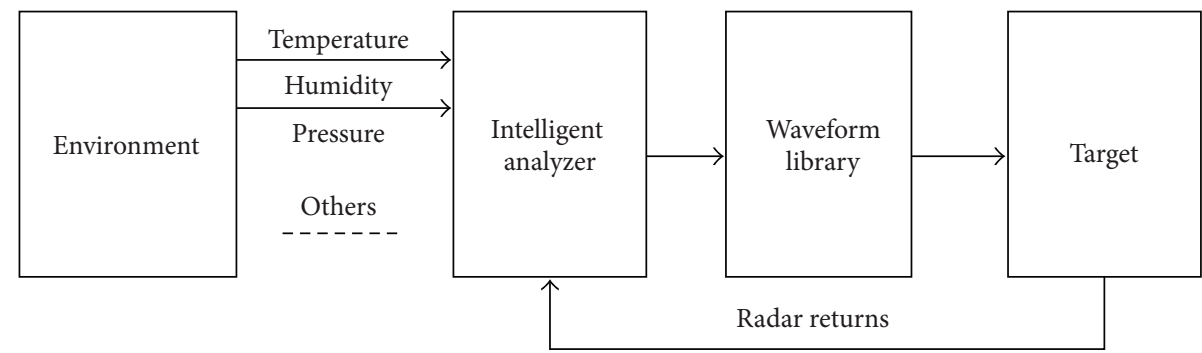

FIGURE 3: Block diagram of waveform library.

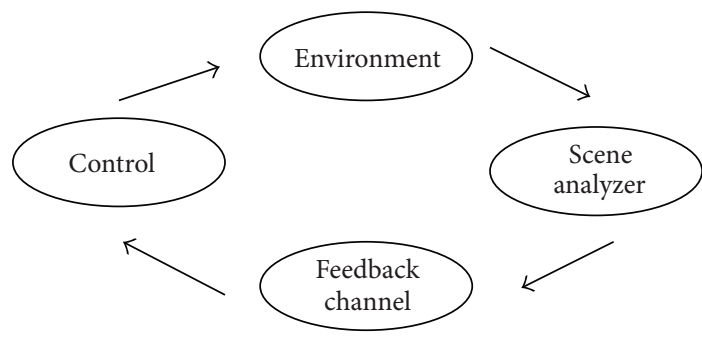

FIGURE 4: Basic signal-processing cycle in cognitive radar.

transmitted energy as possible into the largest mode of the target to maximize the output signal-to-noise ratio (SNR). For estimation task, the optimal radar waveform should allocate the energy between the received signal and the target signature. For other tasks, other performance is required.

Cognitive radar is an intelligent system. In different radar environments, it can synthesize different waveforms. One possible scheme is to make a trade-off among different performances. Efficient algorithms are required in the construction of cognitive radar systems. Such algorithms should provide a flexible framework that can synthesize waveforms that provide different trade-offs between a variety of performance objectives which themselves may also be adapted to the perceived nature of the environment.

We consider two situations of no clutter and clutter and set up their mutual information model, respectively.

Figure 5 is the signal model of a target in which there is no clutter.

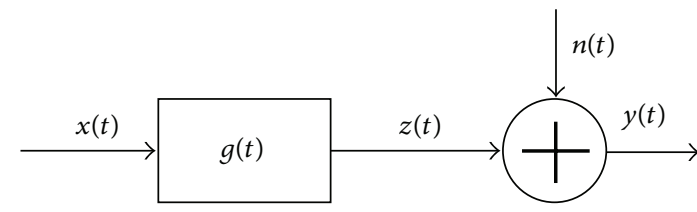

FIGURE 5: Signal model of a target in which there is no clutter.

We want to find the mutual information $I(\mathbf{g}, \mathbf{y} \mid \mathbf{x})$, that is, the mutual information between the random target impulse response and the received radar waveform. Those functions $\mathbf{x}$ that maximize $I(\mathbf{y}, \mathbf{z} \mid \mathbf{x})$ also maximize $I(\mathbf{g}, \mathbf{y} \mid$ $\mathbf{x})$. So we maximize $I(\mathbf{y}, \mathbf{z} \mid \mathbf{x})$ firstly. Assume that target is Rayleigh type and noise is Gaussian type. They are statistically independent.

Assume that $K$ represents frequency domain sampling point, $f_{k}$ is a frequency point. Let $\mathbf{x}_{k}$ correspond to the component of $x(t)$ with frequency components in $F_{k}$, let $\mathbf{z}_{k}$ correspond to the component of $z(t)$ with frequency components in $F_{k}$, and let $\mathbf{y}_{k}$ correspond to the component of $y(t)$ with frequency components in $F_{k}$. So the overall mutual information is

$$
I(\mathbf{y}, \mathbf{z} \mid \mathbf{x})=\sum_{k=1}^{K} I\left(\mathbf{y}_{k}, \mathbf{z}_{k} \mid \mathbf{x}\right) .
$$

Assume that the frequency interval $F_{k}=\left[f_{k}, f_{k}+\Delta f\right]$ is sufficiently small, so for $f \in F_{k}, X(f) \approx X\left(f_{k}\right), Z(f) \approx Z\left(f_{k}\right)$, $Y(f) \approx Y\left(f_{k}\right) . \Delta f$ is the bandwidth. 
Next, we define mutual information. In probability theory and information theory, the mutual information of two random variables is expressed as the dependence of them. Mutual information can be defined from mathematics as

$$
I(Y ; Z)=E_{Y, Z}\left[\log \frac{p(Y, Z)}{p(Y) p(Z)}\right],
$$

where $p(Y, Z)$ is joint probability distribution function, and $p(Y)$ and $p(Z)$ are marginal probability distribution function of $Y$ and $Z$, respectively. Intuitively, mutual information contains the total information of $Y$ and $Z$. Assume that $H(Y)$ represents the marginal entropy of $Y, H(Y \mid Z)$ represents the conditional entropy of $Y$ given $Z$. So the mutual information can also be expressed as

$$
I(Y ; Z)=H(Y)-H(Y \mid Z) .
$$

Since $Z, N$ are statistically independent, so the variance of $Y$ is

$$
\sigma_{Y}^{2}=\sigma_{Z}^{2}+\sigma_{N}^{2}
$$

We will now solve $H(Y)$ and $H(Y \mid Z)$, respectively.

$$
\begin{gathered}
H(Y)=E[\ln p(Y)]=\frac{1}{2} \ln 2 \pi \sigma_{Y}^{2}=\frac{1}{2} \ln 2 \pi\left(\sigma_{Z}^{2}+\sigma_{N}^{2}\right), \\
H(Y \mid Z)=\frac{1}{2} \ln 2 \pi \sigma_{N^{*}}^{2}
\end{gathered}
$$

So the mutual information $I(Y ; Z)$ is given by

$$
\begin{aligned}
I(Y ; Z) & =H(Y)-H(Y \mid Z) \\
& =\frac{1}{2} \ln 2 \pi\left(\sigma_{Z}^{2}+\sigma_{N}^{2}\right)-\frac{1}{2} \ln 2 \pi \sigma_{N}^{2} \\
& =\frac{1}{2} \ln \left(\frac{\sigma_{Z}^{2}+\sigma_{N}^{2}}{\sigma_{N}^{2}}\right) \\
& =\frac{1}{2} \ln \left(1+\frac{\sigma_{Z}^{2}}{\sigma_{N}^{2}}\right) .
\end{aligned}
$$

Referring again to the signals $\mathbf{z}_{k}, \mathbf{y}_{k}, \mathbf{n}_{k}$, and $\mathbf{d}_{k}$ with frequency components confined to the interval $F_{k}=\left[f_{k}, f_{k}+\right.$ $\Delta f]$, we have from the sampling theory that each of the signals can be represented by a sequence of samples taken at a uniform sampling rate of $2 \Delta f$. Since we assume that the spectra $X(f), Z(f)$, and $Y(f)$ are smooth and have a constant value (at least approximately) for all $f \in F_{k}$, the samples of the Gaussian process sampled at a uniform rate $2 \Delta f$ are statistically independent.

The samples $\mathbf{z}_{k}$ are independent, identically distributed random variables with zero mean and variance $\sigma_{Z}^{2}$; we note that the total energy $E_{Z}$ in $\mathbf{z}_{k}$ is

$$
\begin{aligned}
E_{Z} & =\left|Z\left(f_{k}\right)\right|^{2} * 2 \Delta f \\
& =2 \Delta f\left|X\left(f_{k}\right)\right|^{2} \sigma_{G}^{2}\left(f_{k}\right) .
\end{aligned}
$$

Over the time interval $T$, this energy is evenly spread among $2 \Delta f T$ statistically independent samples. Hence, the variance of each sample, $\sigma_{Z}^{2}$, is

$$
\begin{aligned}
\sigma_{Z}^{2} & =\frac{E_{Z}}{2 T \Delta f} \\
& =\frac{2 \Delta f\left|X\left(f_{k}\right)\right|^{2} \sigma_{G}^{2}\left(f_{k}\right)}{2 T \Delta f} \\
& =\frac{\left|X\left(f_{k}\right)\right|^{2} \sigma_{G}^{2}\left(f_{k}\right)}{T} .
\end{aligned}
$$

Similarly, the noise process $\mathbf{n}_{k}$ has total energy $E_{N}$ on the interval $T$ given by

$$
E_{N}=\Delta f P_{N}\left(f_{k}\right) T
$$

This energy is evenly distributed among the $2 T \Delta f$ statistically independent, zero-mean samples of $\mathbf{n}_{k}$. Hence, the variance $\sigma_{N}^{2}$ of each sample is

$$
\sigma_{N}^{2}=\frac{\Delta f P_{N}\left(f_{k}\right) T}{2 T \Delta f}=\frac{P_{N}\left(f_{k}\right)}{2} .
$$

Substituting (8) and (10) into (6), we have that for each sample $Z_{m}$ of $\mathbf{z}_{k}$ and corresponding sample $Y_{m}$ of $\mathbf{y}_{k}$, the mutual information between $Z_{m}$ and $Y_{m}$ is

$$
\begin{aligned}
I\left(Y_{m} ; Z_{m}\right) & =\frac{1}{2} \ln \left(1+\frac{\sigma_{Z}^{2}}{\sigma_{N}^{2}}\right) \\
& =\frac{1}{2} \ln \left[1+\frac{\left|X\left(f_{k}\right)\right|^{2} \sigma_{G}^{2}\left(f_{k}\right) / T}{P_{N}\left(f_{k}\right) / 2}\right] \\
& =\frac{1}{2} \ln \left[1+\frac{2\left|X\left(f_{k}\right)\right|^{2} \sigma_{G}^{2}\left(f_{k}\right)}{P_{N}\left(f_{k}\right) T}\right] .
\end{aligned}
$$

Now these are 2T $\Delta f$ statistically independent sample values for both $\mathbf{z}_{k}$ and $\mathbf{y}_{k}$ in the observation interval $T$. Thus,

$$
\begin{aligned}
I\left(\mathbf{y}_{k}, \mathbf{z}_{k} \mid \mathbf{x}\right) & =2 \Delta f T I\left(Y_{m} ; Z_{m}\right) \\
& =T \Delta f \ln \left[1+\frac{2\left|X\left(f_{k}\right)\right|^{2} \sigma_{G}^{2}\left(f_{k}\right)}{P_{N}\left(f_{k}\right) T}\right] .
\end{aligned}
$$

The overall mutual information is

$$
\begin{aligned}
I(\mathbf{y}, \mathbf{z} \mid \mathbf{x}) & =\sum_{k=1}^{K} I\left(\mathbf{y}_{k}, \mathbf{z}_{k} \mid \mathbf{x}\right) \\
& =\sum_{k=1}^{K} T \Delta f \ln \left[1+\frac{2\left|X\left(f_{k}\right)\right|^{2} \sigma_{G}^{2}\left(f_{k}\right)}{P_{N}\left(f_{k}\right)}\right] .
\end{aligned}
$$

Following we will consider the situation that there is clutter. Figure 6 is the signal model of a target ensemble in ground clutter. Assume that target is Rayleigh type, noise is Gaussian type, and clutter is Rayleigh type. They are statistically independent. 


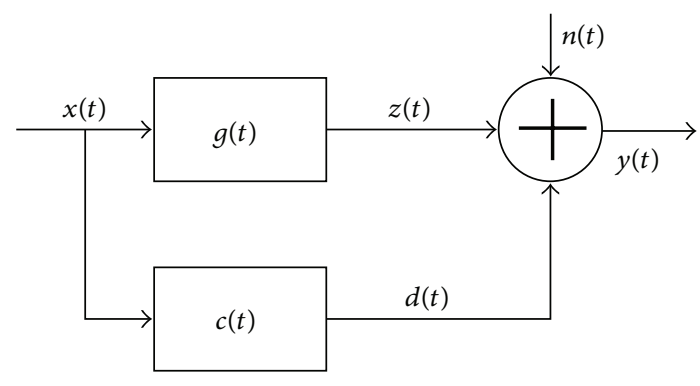

Figure 6: Signal model of a target ensemble in ground clutter.

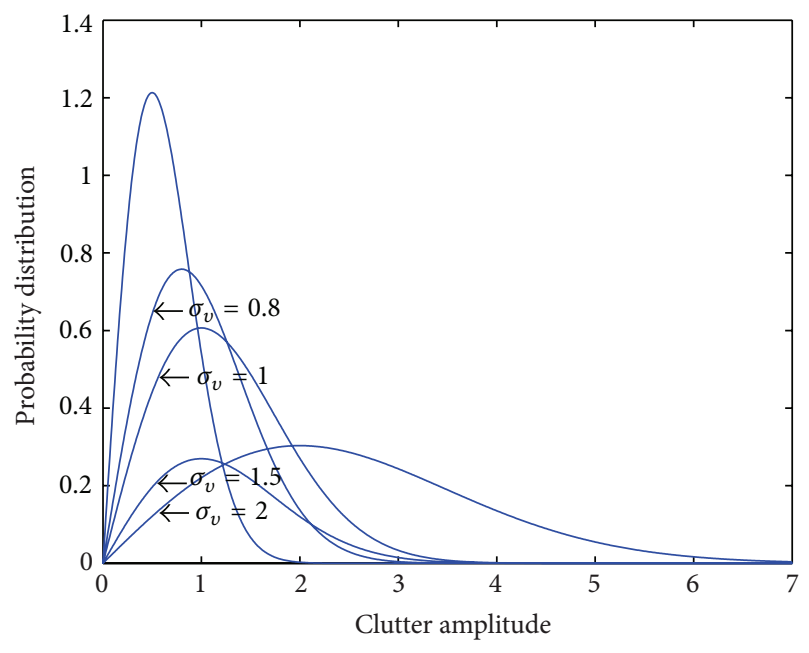

FIgURE 7: Probability distribution of Rayleigh clutter.

We want to find the mutual information $I(\mathbf{g}, \mathbf{y} \mid \mathbf{x})$, that is, the mutual information between the random target impulse response and the received radar waveform. Those functions $\mathbf{x}$ that maximize $I(\mathbf{y}, \mathbf{z} \mid \mathbf{x})$ also maximize $I(\mathbf{g}, \mathbf{y} \mid \mathbf{x})$. So we maximize $I(\mathbf{y}, \mathbf{z} \mid \mathbf{x})$ firstly. Assume that target is Rayleigh type, noise is Gaussian type, and clutter is Rayleigh type.

The probability density distribution function of Rayleigh distribution is

$$
\rho(x)=\frac{x}{\sigma_{V}^{2}} \exp \left(-\frac{x^{2}}{2 \sigma_{V}^{2}}\right), \quad x \geq 0,
$$

where $x$ is clutter amplitude, and $\sigma_{v}$ is standard deviation of clutter. Curve of probability distribution of Rayleigh clutter is in Figure 7. Figure 8 is Rayleigh distribution clutter.

Assume that $K$ represents frequency domain sampling point, $f_{k}$ is a frequency point. Let $\mathbf{x}_{k}$ correspond to the component of $x(t)$ with frequency components in $F_{k}$, let $\mathbf{z}_{k}$ correspond to the component of $z(t)$ with frequency components in $F_{k}$, and let $\mathbf{y}_{k}$ correspond to the component of $y(t)$ with frequency components in $F_{k}$. So the overall mutual information is

$$
I(\mathbf{y}, \mathbf{z} \mid \mathbf{x})=\sum_{k=1}^{K} I\left(\mathbf{y}_{k}, \mathbf{z}_{k} \mid \mathbf{x}\right)
$$

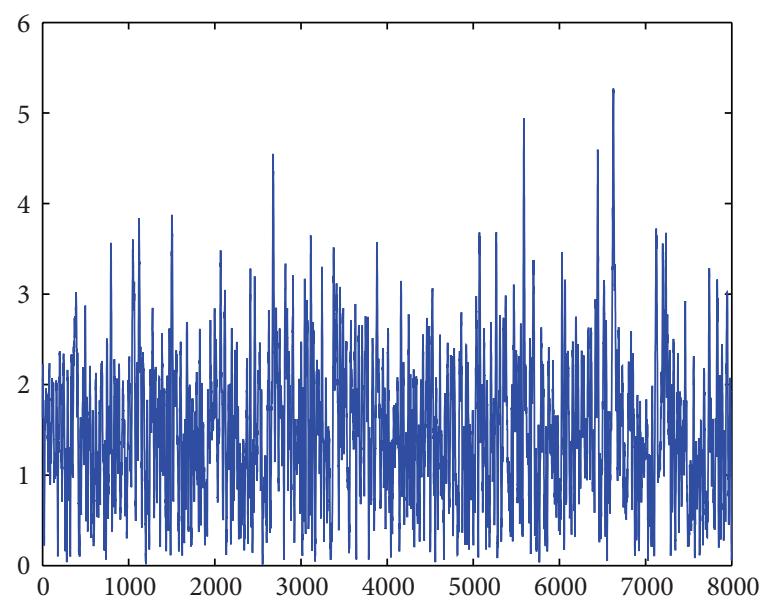

FIGURE 8: Rayleigh distribution clutter.

Assume that the frequency interval $F_{k}=\left[f_{k}, f_{k}+\Delta f\right]$ is sufficiently small, so for $f \in F_{k}, X(f) \approx X\left(f_{k}\right), Z(f) \approx Z\left(f_{k}\right)$, $Y(f) \approx Y\left(f_{k}\right)$, and $D(f) \approx D\left(f_{k}\right) . \Delta f$ is the bandwidth.

Since $Z, N$, and $V$ are statistically independent, so the variance of $Y$ is

$$
\sigma_{Y}^{2}=\sigma_{Z}^{2}+\sigma_{N}^{2}+\sigma_{D}^{2}
$$

We will now solve $H(Y)$ and $H(Y \mid Z)$, respectively, as follows:

$$
\begin{gathered}
H(Y)=E[\ln p(Y)]=\frac{1}{2} \ln 2 \pi \sigma_{Y}^{2}=\frac{1}{2} \ln 2 \pi\left(\sigma_{Z}^{2}+\sigma_{N}^{2}+\sigma_{D}^{2}\right), \\
H(Y \mid Z)=\frac{1}{2} \ln 2 \pi\left(\sigma_{N}^{2}+\sigma_{D}^{2}\right) .
\end{gathered}
$$

So the mutual information $I(Y ; Z)$ is given by

$$
\begin{aligned}
I(Y ; Z) & =H(Y)-H(Y \mid Z) \\
& =\frac{1}{2} \ln 2 \pi\left(\sigma_{Z}^{2}+\sigma_{N}^{2}+\sigma_{D}^{2}\right)-\frac{1}{2} \ln 2 \pi\left(\sigma_{N}^{2}+\sigma_{D}^{2}\right) \\
& =\frac{1}{2} \ln \left(\frac{\sigma_{Z}^{2}+\sigma_{N}^{2}+\sigma_{D}^{2}}{\sigma_{N}^{2}+\sigma_{D}^{2}}\right) \\
& =\frac{1}{2} \ln \left(1+\frac{\sigma_{Z}^{2}}{\sigma_{N}^{2}+\sigma_{D}^{2}}\right) .
\end{aligned}
$$

The total energy $E_{Z}$ in $\mathbf{z}_{k}$ is

$$
\begin{aligned}
E_{Z} & =\left|Z\left(f_{k}\right)\right|^{2} * 2 \Delta f \\
& =2 \Delta f\left|X\left(f_{k}\right)\right|^{2} \sigma_{G}^{2}\left(f_{k}\right) .
\end{aligned}
$$


Over the time interval $T$, this energy is evenly spread among $2 \Delta f T$ statistically independent samples. Hence, the variance of each sample, $\sigma_{Z}^{2}$, is

$$
\begin{aligned}
\sigma_{Z}^{2} & =\frac{E_{Z}}{2 T \Delta f} \\
& =\frac{2 \Delta f\left|X\left(f_{k}\right)\right|^{2} \sigma_{G}^{2}\left(f_{k}\right)}{2 T \Delta f} \\
& =\frac{\left|X\left(f_{k}\right)\right|^{2} \sigma_{G}^{2}\left(f_{k}\right)}{T} .
\end{aligned}
$$

Similarly, the noise process $\mathbf{n}_{k}$ has total energy $E_{N}$ on the interval $T$ given by

$$
E_{N}=\Delta f P_{N}\left(f_{k}\right) T
$$

This energy is evenly distributed among the $2 T \Delta f$ statistically independent, zero-mean samples of $\mathbf{n}_{k}$. Hence, the variance $\sigma_{N}^{2}$ of each sample is

$$
\sigma_{N}^{2}=\frac{\Delta f P_{N}\left(f_{k}\right) T}{2 T \Delta f}=\frac{P_{N}\left(f_{k}\right)}{2} .
$$

Similarly,

$$
\begin{aligned}
E_{V} & =\left|V\left(f_{k}\right)\right|^{2} * 2 \Delta f \\
& =2 \Delta f\left|X\left(f_{k}\right)\right|^{2} \sigma_{D}^{2}\left(f_{k}\right), \\
\sigma_{D}^{2} & =\frac{E_{D}}{2 T \Delta f} \\
& =\frac{2 \Delta f\left|X\left(f_{k}\right)\right|^{2} \sigma_{V}^{2}\left(f_{k}\right)}{2 T \Delta f} \\
& =\frac{\left|X\left(f_{k}\right)\right|^{2} \sigma_{V}^{2}\left(f_{k}\right)}{T} .
\end{aligned}
$$

Substituting (20), (22), and (24) into (18), we have that for each sample $Z_{m}$ of $\mathbf{z}_{k}$ and corresponding sample $Y_{m}$ of $\mathbf{y}_{k}$, the mutual information between $Z_{m}$ and $Y_{m}$ is

$$
\begin{aligned}
I\left(Y_{m} ; Z_{m}\right) & =\frac{1}{2} \ln \left(1+\frac{\sigma_{Z}^{2}}{\sigma_{N}^{2}+\sigma_{D}^{2}}\right) \\
& =\frac{1}{2} \ln \left[1+\frac{\left|X\left(f_{k}\right)\right|^{2} \sigma_{G}^{2}\left(f_{k}\right) / T}{P_{N}\left(f_{k}\right) / 2+\left|X\left(f_{k}\right)\right|^{2} \sigma_{V}^{2}\left(f_{k}\right) / T}\right] \\
& =\frac{1}{2} \ln \left[1+\frac{2\left|X\left(f_{k}\right)\right|^{2} \sigma_{G}^{2}\left(f_{k}\right)}{P_{N}\left(f_{k}\right) T+2\left|X\left(f_{k}\right)\right|^{2} \sigma_{V}^{2}\left(f_{k}\right)}\right] .
\end{aligned}
$$

Now these are $2 T \Delta f$ statistically independent sample values for both $\mathbf{z}_{k}$ and $\mathbf{y}_{k}$ in the observation interval $T$. Thus,

$$
\begin{aligned}
I\left(\mathbf{y}_{k}, \mathbf{z}_{k} \mid \mathbf{x}\right) & =2 \Delta f T I\left(Y_{m} ; Z_{m}\right) \\
& =T \Delta f \ln \left[1+\frac{2\left|X\left(f_{k}\right)\right|^{2} \sigma_{G}^{2}\left(f_{k}\right)}{P_{N}\left(f_{k}\right) T+2\left|X\left(f_{k}\right)\right|^{2} \sigma_{V}^{2}\left(f_{k}\right)}\right] .
\end{aligned}
$$

The overall mutual information is

$$
\begin{aligned}
I & (\mathbf{y}, \mathbf{z} \mid \mathbf{x}) \\
& =\sum_{k=1}^{K} I\left(\mathbf{y}_{k}, \mathbf{z}_{k} \mid \mathbf{x}\right) \\
& =\sum_{k=1}^{K} T \Delta f \ln \left[1+\frac{2\left|X\left(f_{k}\right)\right|^{2} \sigma_{G}^{2}\left(f_{k}\right)}{P_{N}\left(f_{k}\right) T+2\left|X\left(f_{k}\right)\right|^{2} \sigma_{V}^{2}\left(f_{k}\right)}\right] .
\end{aligned}
$$

3. Optimal Waveform Design Using Newton Method and Interior Point Method

Considering the situation that there is no clutter, we can get

$$
I(\mathbf{g}, \mathbf{y} \mid \mathbf{x})=\sum_{k=1}^{K} T \Delta f \ln \left[1+\frac{2\left|X\left(f_{k}\right)\right|^{2} \sigma_{G}^{2}\left(f_{k}\right)}{P_{N}\left(f_{k}\right) T}\right] .
$$

We can write in another form

$$
I(\mathbf{g}, \mathbf{y} \mid \mathbf{x})=T \Delta f \sum_{k=1}^{K} \ln \left(1+\beta\left(f_{k}\right) M_{f_{k}}^{T} \mathbf{R}_{x x}\right),
$$

where $L_{x}$ is the length of discrete transmitted signal, and $\mathbf{R}_{x x}$ is the autocorrelation function of the transmitted signal as follows:

$$
\begin{gathered}
\beta(f)=\frac{2 \sigma_{G}^{2}(f)}{P_{N}(f) T}, \\
M_{f_{k}}=\left[1,2 \cos \left(2 \pi f_{k}\right), 2 \cos \left(4 \pi f_{k}\right), \ldots,\right. \\
\left.2 \cos \left(2 \pi\left(L_{x}-1\right) f_{k}\right)\right]^{T} \quad k=1, \ldots, K, \\
R_{x x}(j)=\sum_{n=0}^{L_{x}-1} x(n+j) x(n), \\
\mathbf{R}_{x x}=\left[R_{x x}(0), R_{x x}(1), \ldots, R_{x x}\left(L_{x}-1\right)\right]^{T} .
\end{gathered}
$$

Following we will consider some constraints.

First, in order to guarantee that radar can detect target, SNR needs to be greater than a certain threshold; that is,

$$
\frac{1}{\sigma_{n}^{2}} E_{\text {signal }} \geq \mathrm{SNR}_{0}
$$

Second, the energy of transmitted signal should be a fixed value; that is,

$$
\int_{0}^{T_{x}} x^{2}(t) d t=E_{x}
$$

Third, the power of transmitted signal should be greater than a certain threshold; that is,

$$
\int_{f}^{f_{0}+W}|X(f)|^{2} d f \geq P_{x} .
$$


Finally, the PSD of transmitted signal should be nonnegative for all frequencies; that is,

$$
S_{x x}(f) \geq 0 \text {. }
$$

Let the four constraints convert the constraint that contains $\mathbf{R}_{x x}$, then the optimization problem can be expressed as

$$
\begin{array}{ll}
\min _{\mathbf{R}_{x x}} & -\sum_{k=1}^{K} \ln \left(1+\widetilde{\beta}\left(f_{k}\right) \widetilde{M}_{f_{k}}^{T} \widetilde{\mathbf{R}}_{x x}\right), \\
\text { s.t. } & -\left[\widetilde{G}^{T}, \mathbf{0}_{1 \times\left(L_{x}-L_{g}\right)}\right] \widetilde{\mathbf{R}}_{x x} \leq \mathbf{g}^{T} \mathbf{g} E_{x}-\frac{\sigma_{n}^{2}}{T_{s}^{2}} S N R_{0} \\
& -\widetilde{p}^{T} \widetilde{\mathbf{R}}_{x x} \leq W E_{x}-P_{x} \\
& -\widetilde{M}_{f_{k}}^{T} \widetilde{\mathbf{R}}_{x x} \leq E_{x},
\end{array}
$$

where

$$
\begin{aligned}
& \mathbf{R}_{x x}=\left[R_{x x}[0], \widetilde{\mathbf{R}}_{x x}\right] \text {, } \\
& \tilde{\beta}\left(f_{k}\right)=\frac{2 \sigma_{g}^{2}\left(f_{k}\right)}{\sigma_{n}^{2} T+2 \sigma_{n}^{2}\left(f_{k}\right) E_{x}}, \\
& \widetilde{G}^{T}=2 \mathbf{g}^{T}\left[\overleftarrow{L^{1}}\left(\mathbf{g}^{T}\right)^{T}, \ldots, \overleftarrow{L}^{L_{g-1}}\left(\mathbf{g}^{T}\right)^{T}\right] \\
& \mathbf{g}=\left[g(1), g(2), \ldots, g\left(L_{g}\right)\right]^{T}, \\
& \widetilde{p}=\left[\frac{1}{\pi}\left(\left.(\sin 2 \pi)\right|_{f_{0}} ^{f_{0}+W}\right), \ldots, \frac{1}{\left(L_{x}-1\right) \pi}\right. \\
& \left.\times\left(\left.\sin 2 \pi\left(L_{x}-1\right) f\right|_{f_{0}} ^{f_{0}+W}\right)\right]^{T}, \\
& \widetilde{M}_{f_{k}}=\left[2 \cos \left(2 \pi f_{k}\right), 2 \cos \left(4 \pi f_{k}\right), \ldots,\right. \\
& \left.2 \cos \left(2 \pi\left(L_{x}-1\right) f\right)\right]^{T} .
\end{aligned}
$$

Writing the optimization problem in a simple form, we can get

$$
\begin{array}{ll}
\min _{x} & -\sum_{k=1}^{K} \ln \left(1+c_{k}^{T} x\right), \\
\text { s.t. } & a_{1}^{T} x \leq b_{1} \\
& a_{2}^{T} x \leq b_{2} \\
& a_{i+2}^{T} x \leq b_{i+2}, \quad i=1, \ldots, K,
\end{array}
$$

where

$$
\begin{gathered}
x=\widetilde{\mathbf{R}}_{x x}, \\
c_{k}^{T}=\widetilde{\beta}\left(f_{k}\right) \widetilde{M}_{f_{k}}^{T}, \quad k=1, \ldots, K, \\
a_{1}^{T}=-\left[\widetilde{G}^{T}, \mathbf{0}_{1 \times\left(L_{x}-L_{g}\right)}\right],
\end{gathered}
$$

$$
\begin{gathered}
b_{1}=\mathbf{g}^{T} \mathbf{g} E_{x}-\frac{\sigma_{n}^{2}}{T_{s}^{2}} \mathrm{SNR}_{0}, \\
a_{2}^{T}=-\widetilde{p}^{T}, \\
b_{2}=W E_{x}-P_{x}, \\
a_{k+2}^{T}=-\widetilde{M}_{f_{k}}^{T}, \quad k=1, \ldots, K, \\
b_{k+2}=E_{x}, \quad k=1, \ldots, K .
\end{gathered}
$$

Following we will use Newton method and interior point method to solve the optimization problem. Using a logbarrier function, the optimization can be changed into the following form:

$$
\min _{x} f(x)=-t_{\mathrm{br}} \sum_{k=1}^{K} \ln \left(1+c_{k}^{T} x\right)-\sum_{i=1}^{K+2} \ln \left(b_{i}-a_{i}^{T} x\right) .
$$

The gradient and Hessian of $f(x)$ can be calculated by

$$
\begin{gathered}
\nabla f(x)=-t_{\mathrm{br}} \sum_{k=1}^{K} \frac{1}{1+c_{k}^{T}} c_{k}+\sum_{i=1}^{K+2} \frac{1}{b_{i}-a_{i}^{T} x} a_{i}, \\
\nabla^{2} f(x)=t_{\mathrm{br}} \sum_{k=1}^{K} \frac{1}{\left(1+c_{k}^{T}\right)^{2}} c_{k} c_{k}^{T}-\sum_{i=1}^{K+2} \frac{1}{\left(b_{i}-a_{i}^{T} x\right)^{2}} a_{i} a_{i}^{T} .
\end{gathered}
$$

Using Newton method to find the optimal solution, Newton step size and Newton descent are needed to calculate. The formula of Newton step size is

$$
\begin{aligned}
t_{\mathrm{nt}} & =-\nabla^{2} f(x)^{-1} \nabla f(x) \\
& =\frac{\sum_{i=1}^{K+2}\left(1 /\left(b_{i}-a_{i}^{T} x\right)\right) a_{i}-t_{\mathrm{br}} \sum_{k=1}^{K}\left(1 /\left(1+c_{k}^{T}\right)\right) c_{k}}{\sum_{i=1}^{K+2}\left(1 /\left(b_{i}-a_{i}^{T} x\right)^{2}\right) a_{i} a_{i}^{T}-t_{\mathrm{br}} \sum_{k=1}^{K}\left(1 /\left(1+c_{k}^{T}\right)^{2}\right) c_{k} c_{k}^{T}} .
\end{aligned}
$$

The formula of Newton descent is

$$
\lambda(x)=\sqrt{-\left(\nabla f(x)^{T} \nabla^{2} f(x)^{-1} \nabla f(x)\right)} .
$$

Then we will use interior point method to solve the optimization problem. Interior point method contains double loop. The outer loop step size is $t_{\mathrm{br}}$, and the inner loop (Newton loop) step size is $t_{\mathrm{nt}}$. The algorithm can be described as follows:

(1) given an initial value $t_{\mathrm{br} 0}$, execute Newton loop;

(2) given an initial value $x_{0} \in \operatorname{dom} f$, and allowable error $\varepsilon_{\text {nt }}>0$;

(3) calculate Newton step size

$$
t_{\mathrm{nt}}=-\nabla^{2} f(x)^{-1} \nabla f(x) ;
$$

(4) calculate Newton descent

$$
\lambda(x)=\sqrt{-\left(\nabla f(x)^{T} \nabla^{2} f(x)^{-1} \nabla f(x)\right)} ;
$$




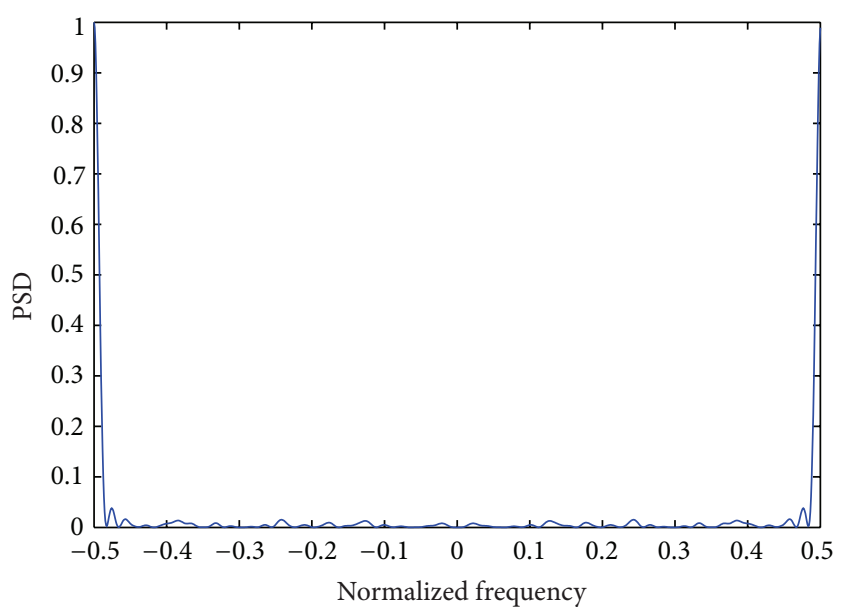

FIGURE 9: PSD of Rayleigh target impulse.

(5) when $\lambda^{2} / 2 \leq \varepsilon_{\mathrm{nt}}$, stop Newton loop and return optimal point $x_{0}$; otherwise, given initial value $t_{\mathrm{nt} 0}$, renew variable $x_{0}=x_{0}+t_{\mathrm{nt} 0} * t_{\mathrm{nt}}$;

(6) for the $x_{0}$ Newton loop returns, certify in the outer loop. If $M / t_{\mathrm{br}} \leq \varepsilon_{\mathrm{br}}$, the $x_{0}$ Newton loop returns are the global optimal point; otherwise, calculate $t_{\mathrm{br}}=$ $\mu t_{\mathrm{br}}$ and execute Newton loop.

For the situation there is clutter, and we can get

$$
\begin{aligned}
& I(\mathbf{g}, \mathbf{y} \mid \mathbf{x}) \\
& \quad=\sum_{k=1}^{K} T \Delta f \ln \left[1+\frac{2\left|X\left(f_{k}\right)\right|^{2} \sigma_{G}^{2}\left(f_{k}\right)}{P_{N}\left(f_{k}\right) T+2\left|X\left(f_{k}\right)\right|^{2} \sigma_{V}^{2}\left(f_{k}\right)}\right] .
\end{aligned}
$$

Using the previous method, we can solve the optimization problem when there is clutter.

\section{Simulations}

We consider a point target in the radar's surveillance region with a known impulse response. Suppose that the frequency of the signal is normalized to be $(0,1)$. In order to satisfy the Shannon sampling theorem, the sampling frequency is set to 2. The lengths of target impulse response and waveform vector are 63 and 63, respectively. The energy constraint is 1 , and power percentage is 0.9 . This frequency interval is divided into 2048 non-overlapping frequency bins. The tolerance of Newton method is $10^{-5}$. The noise variance is 0.1 . The SNR threshold is $-5 \mathrm{~dB}$. Tolerance of Newton method and barrier method are $10^{-5}$ and $10^{-5}$, respectively. The step size increment factor for the barrier method loop is 5. Initial step size for outer loop and inner loop is 5 and 1 , respectively. Parameters in backtracking line search method are 0.3 and 0.7 , respectively. We use Rayleigh-type radar signature in this paper.

Figure 9 is PSD of Rayleigh target impulse. Figure 10 is PSD of transmitted waveform. Figure 11 is the superposition of PSD of Rayleigh target impulse and transmitted waveform

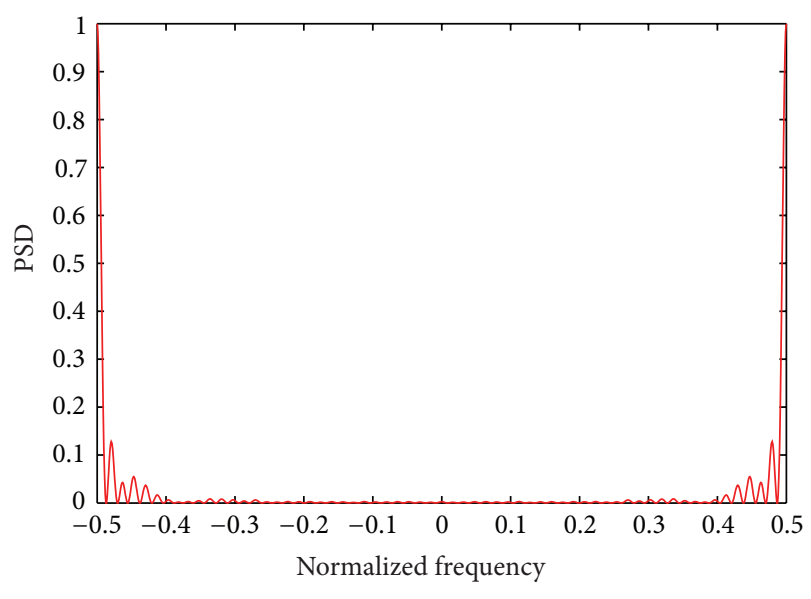

FIGURE 10: PSD of transmitted waveform.

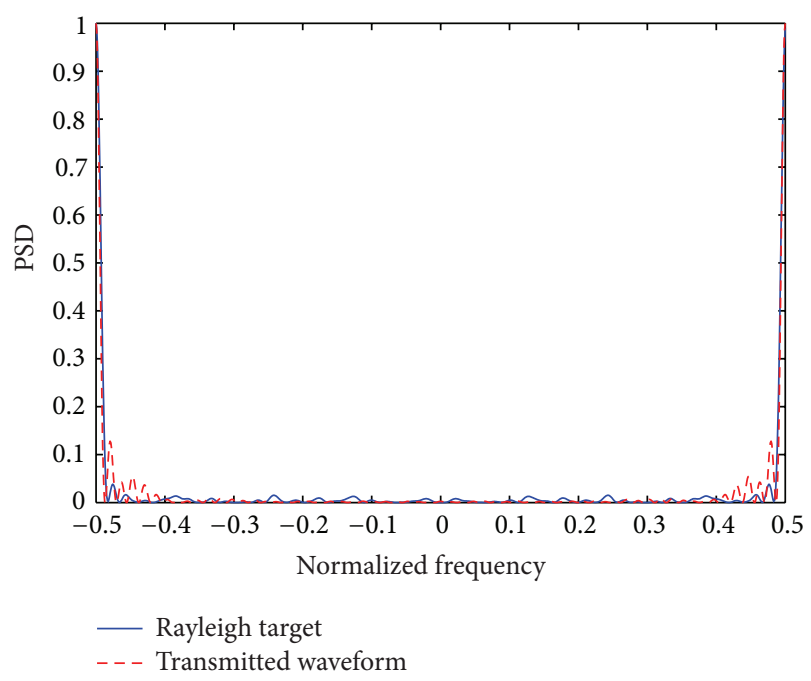

FIGURE 11: Superposition of PSD of Rayleigh target impulse and transmitted waveform (no clutter).

(no clutter). The figure shows that the optimal radar waveforms will spread its energy among most of the spectral peaks of the target response. When mutual information reaches maximum, the peak of PSD of transmitted waveform changes with the peak of PSD of target impulse. So in order to maximize the mutual information in the waveform design of cognitive radar, we should transmit the waveform whose peak of PSD changes with that of target impulse.

Figure 12 is mutual information for all central points. It can be seen that when the central point can accurately approximate the optimal point, the mutual information tends to reach the maximum. The mutual information is greater than that of Bell proposed in [15].

Figure 13 is superposition of PSD of Rayleigh target impulse and transmitted waveform (in clutter). The figure shows that the optimal radar waveforms will spread its energy among most of the spectral peaks of the target response. When mutual information reaches maximum, the peak of PSD of transmitted waveform changes with the peak of PSD 




FIGURE 12: Mutual information for all central points.

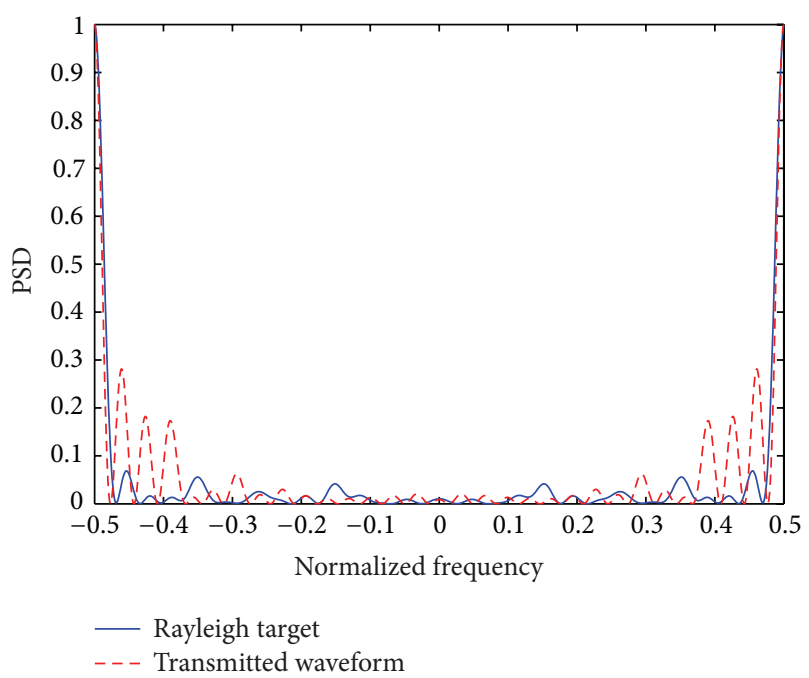

FIGURE 13: Superposition of PSD of Rayleigh target impulse and transmitted waveform (no clutter).

of target impulse. However, as a result of clutter influence, the trend level of the peak of PSD of transmitted waveform to that of target impulse in clutter is weak than no clutter. So the design of transmitted waveform should consider the influence of clutter. The quantitative analysis of clutter to the trend level is necessary.

Figure 14 is mutual information for all central points. It can be seen that when the central point can accurately approximate the optimal point, the mutual information tends to reach the maximum. The mutual information is also greater than that of Bell proposed in [15]. However, the mutual information is lower than that in Figure 11 due to the influence of clutter. So we should consider the quantitative analysis of clutter to the mutual information.

\section{Conclusions}

Cognitive radar can optimally decide or select the radar waveform for next transmission based on the observations of

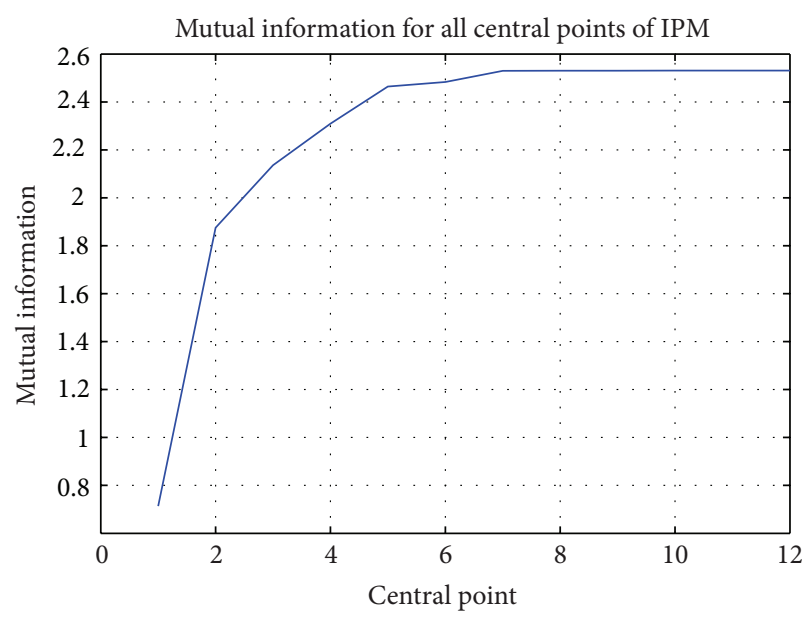

FIGURE 14: Mutual information for all central points.

past radar returns. Adaptive waveform design is an important problem in cognitive radar. In this paper, we propose mutual information model of adaptive waveform design, which can convert the problem of adaptive waveform design into the problem of optimization. We consider two situations of no clutter and clutter and use Newton method and interior point method to solve the optimization problem. From the simulation results, we can see that using the IPM, the mutual information tends to reach the maximum when the central point can accurately approximate the optimal point, and the mutual information is greater than that proposed before. In the next step we should consider the quantitative analysis of clutter to the trend level and the mutual information.

\section{Acknowledgments}

This work was supported by the National Natural Science Foundation of China, under Grant no. 61004052 and 61104005. It was also supported by the Fundamental Research Funds for the Central Universities, under Grant no. N110323005.

\section{References}

[1] S. Haykin, "Cognitive radar: a way of the future," IEEE Signal Processing Magazine, vol. 23, no. 1, pp. 30-40, 2006.

[2] S. Haykin, "Cognition is the key to the next generation of radar systems," in Proceedings of the Digital Signal Processing Workshop and 5th IEEE Signal Processing Education Workshop (DSP/SPE '09), pp. 463-467, IEEE Press, 2009.

[3] I. Arasaratnam and S. Haykin, "Cubature kalman filters," IEEE Transactions on Automatic Control, vol. 54, no. 6, pp. 1254-1269, 2009.

[4] S. Haykin, Y. B. Xue, and T. Davidson, Optimal WaveForm Design For Cognitive Radar, in Asilomar Conference, IEEE Press, 2008.

[5] Y. Yang and R. S. Blum, "Waveform design for MIMO radar based on mutual information and minimum mean-square error estimation," in Proceedings of the 40th Annual Conference on Information Sciences and Systems, pp. 111-116, IEEE Press, 2006. 
[6] N. A. Goodman, R. P. Venkata, and A. M. Neifeld, "Adaptive waveform design and sequential hypothesis testing for target recognition with active sensors," IEEE Journal of Selected Topics in Signal Processing, vol. 1, no. 1, pp. 105-1132, 2007.

[7] S. P. Sira, A. Papandreou-Suppappola, and D. Morrell, "Dynamic configuration of time-varying waveforms for agile sensing and tracking in clutter," IEEE Transactions on Signal Processing, vol. 55, no. 7, part 1, pp. 3207-3217, 2007.

[8] Y. Yang and R. S. Blum, "MIMO radar waveform design based on mutual information and minimum mean-square error estimation," IEEE Transactions on Aerospace and Electronic Systems, vol. 43, no. 1, pp. 330-343, 2007.

[9] A. Leshem, O. Naparstek, and A. Nehorai, "Information theoretic adaptive radar waveform design for multiple extended targets," IEEE Journal of Selecte Topics in Signal Processing, vol. 1, no. 1, pp. 42-55, 2007.

[10] R. A. Romero and N. A. Goodman, "Waveform design in singledependent interference and application to target recognition with multiple transmissions," IET Radar, Sonar and Navigation, vol. 3, no. 4, pp. 328-340, 2009.

[11] K. S. Kwon, "Waveform design methods for piezo inkjet dispensers based on measured menicus motion," Journal of Microelectromechanical Systems, vol. 18, no. 5, pp. 1118-1125, 2009.

[12] D. J. Kershaw and R. J. Evans, "Waveform selective probabilistic data association," IEEE Transactions on Aerospace and Electronic Systems, vol. 33, no. 4, pp. 1180-1188, 1997.

[13] S. P. Sira and D. Cochran, "Adaptive waveform design for improved detection of low-RCS targets in heavy sea clutter," IEEE Journal of Selected Topics in Signal Processing, vol. 1, no. 1, pp. 56-66, 2007.

[14] C. Rago, P. Willett, and Y. Bar-Shalom, "Detecting-tracking performance with combined waveforms," IEEE Transactions on Aerospace and Electronic Systems, vol. 34, no. 2, pp. 612-624, 1998.

[15] M. R. Bell, "Information theory and radar waveform design," IEEE Transactions on Information Theory, vol. 39, no. 5, pp. 1578-1597, 1993. 


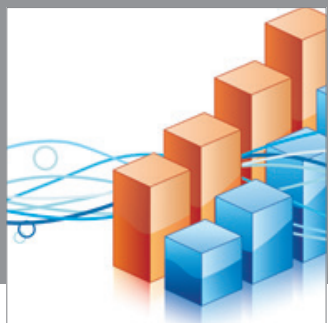

Advances in

Operations Research

mansans

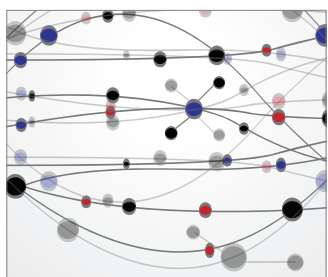

The Scientific World Journal
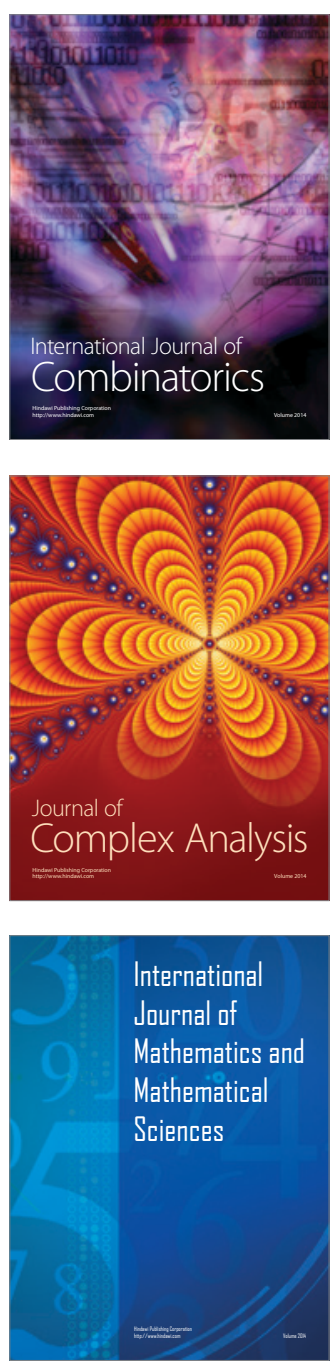
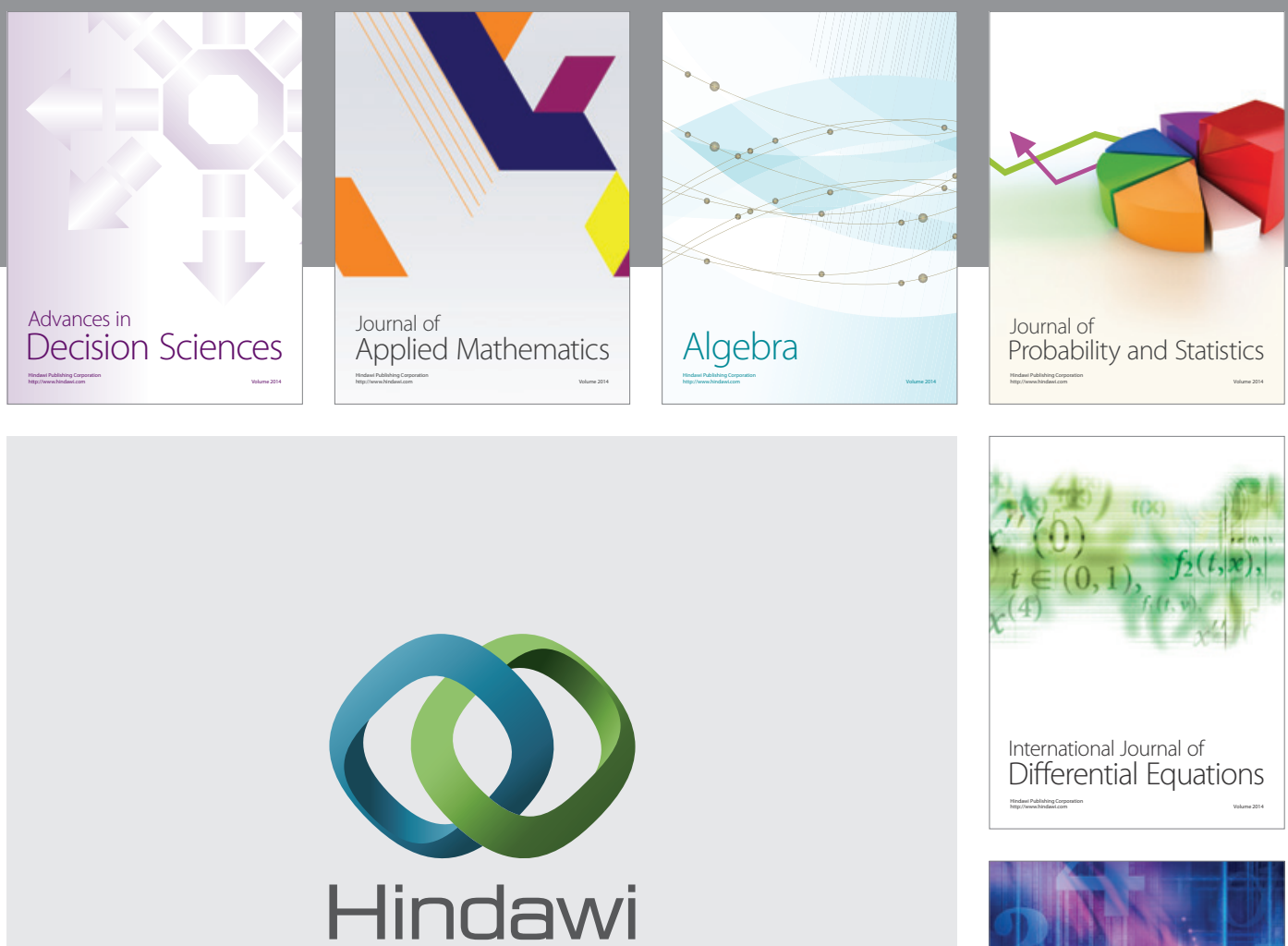

Submit your manuscripts at http://www.hindawi.com


Journal of

Function Spaces

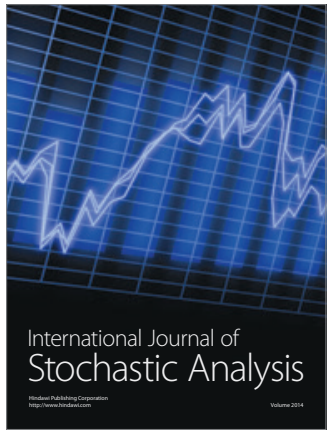

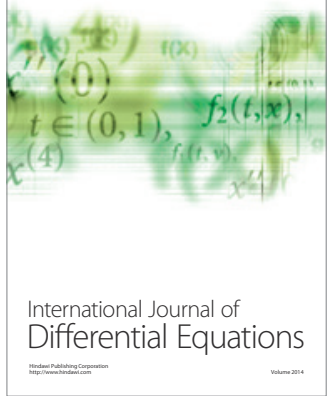
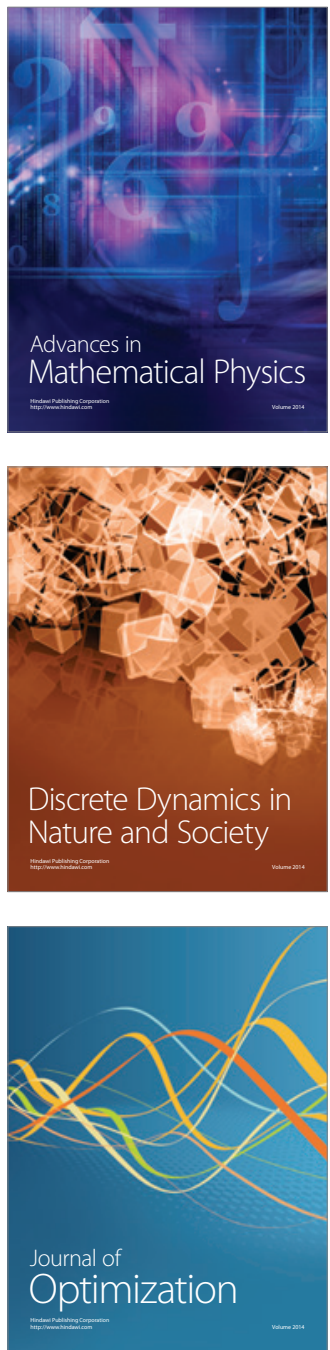